\title{
Impact of ionospheric irregularity on SBAS integrity: spatial threat modeling and improvement
}

\author{
BAO Junjie, LI Rui, LIU Pan*, and HUANG Zhigang \\ School of Electronic and Information Engineering, Beihang University, Beijing 100191, China
}

\begin{abstract}
The ionosphere, as the largest and least predictable error source, its behavior cannot be observed at all places simultaneously. The confidence bound, called the grid ionospheric vertical error (GIVE), can only be determined with the aid of a threat model which is used to restrict the expected ionospheric behavior. However, the spatial threat model at present widespread used, which is based on fit radius and relative centroid metric (RCM), is too conservative or the resulting GIVEs will be too large and will reduce the availability of satellite-based augmentation system (SBAS). In this paper, layered two-dimensional parameters, the vertical direction double RCMs, are introduced based on the spatial variability of the ionosphere. Comparing with the traditional threat model, the experimental results show that the user ionospheric vertical error (UIVE) average reduction rate reaches $16 \%$. And the $95 \%$ protection level of conterminous United States (CONUS) is $28 \%$, even under disturbed days, which reaches about $5 \%$ reduction rates. The results show that the system service performance has been improved better.
\end{abstract}

Keywords: ionospheric delay, spatial threat model, relative centroid metric (RCM), user ionospheric vertical error (UIVE).

DOI: $10.21629 / J S E E .2018 .05 .03$

\section{Introduction}

Ionospheric delay, as one of the main error sources of global navigation satellite system (GNSS), has a considerable influence on the navigation capability of single frequency users $[1-6]$. The main studies of the ionospheric grid algorithm are divided into two parts. The first is ionospheric delay corrections estimation method, which uses the single-layer shell model hypothesis to calculate ionospheric grid to analyze the ionospheric characteristics between the reference stations and satellites, and estimate the user's ionospheric vertical delays and vertical errors. Scholars used a type of minimum mean square estimator technology adapted to spatial data, named planar Kriging

\footnotetext{
Manuscript received April 26, 2017.

*Corresponding author.

This work was supported by the National Natural Science Foundation of China (41304024).
}

[7-14]. However, because this method hardly considers the ionospheric electron density in the vertical direction, some scholars proposed the second method, the multi-layer model combined with the Kriging method and tomography technology to improve the positioning accuracy, named the extended Kriging algorithm [15-17].

However, when ionosphere becomes irregularity, the actual ionospheric delay no longer conforms to the established estimation model and the ionospheric pierce points (IPPs) numbers may be reduced or with non-uniformity distribution, termed under-sampled. This condition will lead to the large ionospheric delay estimation error and integrity faults in wide area augmentation system (WAAS). Therefore, we focus on another study of ensuring the integrity of the user by the inflated models and spatial threat models [18-21]. The inflated model mainly uses the inflation factor, increases the grid ionospheric vertical error (GIVE) value of storm period, and overcomes the influence caused by the ionospheric disturbance. With the phenomenon of under-sampled, the spatial threat model is established to solve it [22-24].

The original ionospheric spatial threat model is a simple function of the planar fit radius and the maximum deviations from planarity of each function is used as the worst-case deviation that a specific radius could ever encounter. However, some investigations are found that the large threats are associated with the distributions of IPPs, which leads to a second metric to characterize the offset of the centroid of the IPP distribution in terms of the overall radius of the planar fit, namely relative centroid metric (RCM).

At present, the spatial threat model which is a function of fit radius $\left(R_{\mathrm{fit}}\right)$ and RCM are currently used in WAAS [25-27]. Unfortunately, RCM cannot describe the actual geometric distribution of the pierce points accurately, especially those have small elevation angle, which leads to the conservative estimation of the GIVE value. To cope with this problem, in this paper, the double RCMs are pro- 
posed to establish the layered two-dimensional (L2D) spatial threat model based on extended Kriging. RCM of different heights is calculated to further describe the distribution of IPPs.

This paper is organized as follows. Firstly, the basic estimation method is introduced to calculate delays and GIVE of ionospheric grid point (IGP). Secondly, the L2D spatial threat model based on the double RCMs and fit radius are established with the data of the storm period, and compared with the accuracy of the 2D and L2D threat model. Then, ionospheric delay measurements in the storm period and quiet period are used to analyze the user grid correction performance and system service performance. Finally, we offer a brief summary of the paper's key points.

\section{Ionospheric spatial threat model}

\subsection{Estimation method of IGP corrections}

Based on the measurements collected in a network of reference stations, WAAS computes and broadcasts an estimate of the vertical delay corrections at each IGP, include the grid ionospheric vertical delay (GIVD) and a safetycritical integrity bound GIVE, to allow the user to correct the error caused by the ionosphere delay. For each satelliteto-receiver raypath, the ionospheric slant delay is a linear combination of sample values in a certain range. Hypothesis: there are $n$ measurements around the grid to meet the requirement, the ionospheric delay expression is as follows:

$$
\hat{I}_{\text {unknown }}=\sum_{i=1}^{n} \lambda_{i} \tilde{I}_{\text {meas }, i} .
$$

Here, the extended Kriging algorithm uses a $p$ layers model instead of the single-layer thin shell, the $i$ th measured in the propagation path and ionospheric estimation variance is equivalent [15] to

$$
\begin{gathered}
I_{\text {slant }, i}=\sum_{k=1}^{p} \varphi_{k} \cdot\left(m+r_{k}\left(x_{k, i}\right)\right) \cdot o b_{k, i} \\
\hat{\sigma}_{\text {Kriging }}^{2}=\boldsymbol{\Lambda}^{\mathrm{T}} \boldsymbol{C}\left(x_{k}, x_{l}\right) \boldsymbol{\Lambda}-2 \boldsymbol{\Lambda}^{\mathrm{T}} \boldsymbol{C}\left(x, x_{k}\right)+\boldsymbol{C}(x, x)
\end{gathered}
$$

where variable $m$ can be interpreted as the mean value and $r_{k}(x)$ is defined as a multi-dimensional Gaussian random field. $x_{k, i}$ designates the IPP location of the $i$ th measurement on the $k$ th layer and $o b_{k, i}$ is the obliquity factor. The purpose of this factor is to convert vertical delay to slant delay.

The fitting coefficient is weighted by using the pierce point delay within the fit radius on the grid points, we can write, after some algebra, that

$$
\boldsymbol{\Lambda}=\left(\boldsymbol{W}-\boldsymbol{W} \boldsymbol{G}\left(\boldsymbol{G}^{\mathrm{T}} \boldsymbol{W} \boldsymbol{G}\right)^{-1} \boldsymbol{G}^{\mathrm{T}} \boldsymbol{W}\right) \boldsymbol{C}\left(x, x_{k}\right)+
$$

$$
\boldsymbol{W} \boldsymbol{G}\left(\boldsymbol{G}^{\mathrm{T}} \boldsymbol{W} \boldsymbol{G}\right)^{-1} X_{\text {unknown }}
$$

where

$$
\begin{gathered}
\boldsymbol{C}\left(x, x_{k}\right)=\operatorname{cov}\left(I_{\text {unknown }}, I_{i}\right) \\
\boldsymbol{W}=\left(\operatorname{cov}\left(I_{i}, I_{j}\right)\right)^{-1}= \\
\left(\sum_{k, l} \varphi_{k} \varphi_{l} o b_{k, i} o b_{l, j} \operatorname{cov}\left(r_{k}\left(x_{k, i}\right), r_{k}\left(x_{l, j}\right)\right)\right)^{-1} \\
G_{i}=\sum_{k=1}^{p} \varphi_{k} \cdot o b_{k, i} \\
X_{\text {unknown }}=\sum_{k=1}^{p} \varphi_{k} \cdot o b_{k, \text { unknown. }}
\end{gathered}
$$

$\boldsymbol{W}$ is the inverse of the covariance corresponding to the measurements, $\boldsymbol{C}\left(x, x_{k}\right)$ and $\boldsymbol{G}_{i}$ is an $n$ by 1 vector.

In WAAS, the GIVE of grid points is used to characterize the GIVD estimation accuracy and provide error bounds for user's local ionospheric behavior. The GIVE with consideration of ionospheric irregularity is finally computed as (6) and (7).

$$
\begin{gathered}
\text { GIVE }=\kappa_{99.9 \%} \sigma_{\mathrm{GIVE}} \\
\sigma_{\text {GIVE }}^{2}=R_{\text {irreg }}^{2} \hat{\sigma}_{\text {IGP }}^{2}+\sigma_{\text {undersampled }}^{2}
\end{gathered}
$$

According to (7), the GIVE is mainly composed of two components: one is a term relating to inflation factor which indicates the level of the actual ionospheric delay does not conform to the assumption ionospheric delay model. The covariance expansion model was established to correct the random covariance function of ionospheric vertical delay. The specific expressions are as follows:

$R_{\text {irreg }}^{2}=\alpha_{n} \boldsymbol{I}_{\text {meas }}^{\mathrm{T}}\left(\boldsymbol{W}-\boldsymbol{W} \boldsymbol{G}\left(\boldsymbol{G}^{\mathrm{T}} \boldsymbol{W} \boldsymbol{G}\right)^{-1} \boldsymbol{G}^{\mathrm{T}} \boldsymbol{W}\right) \boldsymbol{I}_{\text {meas }}$

where $\alpha_{n}$ can be calculated by the probability which is assigned to the ionosphere part of integrity threat probability (see (9)), and stored to conveniently calculate. A detailed derivation of $R_{\text {irreg }}^{2}$ can be found in the work of Blanch [28].

$$
P=\frac{2}{\pi} \int_{\varphi=0}^{\frac{\pi}{2}}\left(K^{2} \frac{\alpha_{n}}{\sin ^{2} \varphi}+1\right)^{-\frac{n-3}{2}} \mathrm{~d} \varphi
$$

Another component of CIVE is related to the variance of the spatial threat model which indicates the potential threat caused by the irregularity region under-sampled. The next subsection gives a more detailed description of the ionospheric spatial threat method and of deficiency and solutions for it.

\subsection{Calculation of ionospheric spatial threat}

Generally, in the ionospheric grid algorithm, if the estimated value of GIVD is more accurate, the GIVE value is 
smaller, and vice versa, the GIVE is greater. However, this theory assumes that the ionosphere is well sampled. Unfortunately, the fact is that the ionospheric disturbance and the IPPs under-sampling will make the GIVD estimation accuracy decrease. The worst case is that the user IPP is located in the ionospheric irregularity region, as shown in Fig. 1, where red plots represent IPPs observed from a user. Green IPPs are used for the estimation of the status of the IGP. If so, the user may suffer the misleading information which comes from a large ionospheric delay bias. Thus, this case results in the development of the threat model.

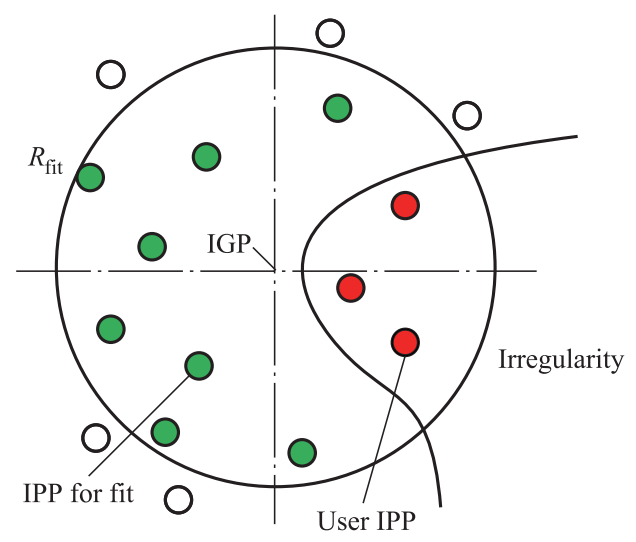

Fig. 1 IPPs under-sampled threat

Here, "threat" is defined as a class of ionospheric behavior that could lead to an integrity failure and the threat model is a methodology to put a hard bound on the possible threats present in archived real ionospheric data [29]. As a consequence, how to protect the user from the delay estimation error caused by the ionospheric irregularity, the solution of this question is that the value of the spatial threat model based on the IPPs distribution around the IGP must be added to the GIVE value, as shown in (7).

According to the definition of satellite-based augmentation system (SBAS), GIVE should envelop the real ionospheric delay estimation error and then it must satisfy the following relationship:

$$
\left|I_{\kappa}-\hat{I}_{\kappa}\right|^{2}=K_{\text {undersampled }}^{2}\left[\hat{\sigma}_{\kappa}^{2}+\sigma_{\text {undersampled }, \kappa}^{2}\right]
$$

where $I_{\kappa}$ and $\hat{I}_{\kappa}$ are expressed as observations and estimates of the vertical delay, respectively. $\hat{\sigma}_{\kappa}^{2}$ is the ionospheric estimation variance. $K_{\text {undersampled }}$ is the upper bound for Gaussian distribution. According to (10), we can obtain the value of $\sigma_{\text {undersampled }}^{2}$.

$$
\sigma_{\text {undersampled }}^{2}=\frac{\left|I_{\kappa}-\hat{I}_{\kappa}\right|^{2}}{K_{\text {undersampled }}^{2}}-\tilde{\sigma}_{k}^{2}
$$

The calculation of $\sigma_{\text {undersampled, } \kappa}^{2}$ should take into account the fact that the distribution of all IPPs is adjacent to the grid point in the threat area, and the changes within a certain time interval. The calculation formula of the $2 \mathrm{D}$ spatial threat model as follows:

$$
\begin{gathered}
\sigma_{\text {undersampled }}^{\text {raw }}\left(R_{\mathrm{fit}}, \mathrm{RCM}\right)= \\
\max _{\text {over }, T}\left[\sqrt{\frac{\left|I_{\kappa}-\hat{I}_{\kappa}\right|^{2}}{K_{\text {undersampled }}^{2}}-\hat{\sigma}_{\kappa}^{2}}\right] .
\end{gathered}
$$

As shown in Fig. 2, where the RCM is the ratio of the distance between the centroid of IPPs to IGP and the fit radius $(0<\mathrm{RCM} \leqslant 1)$.

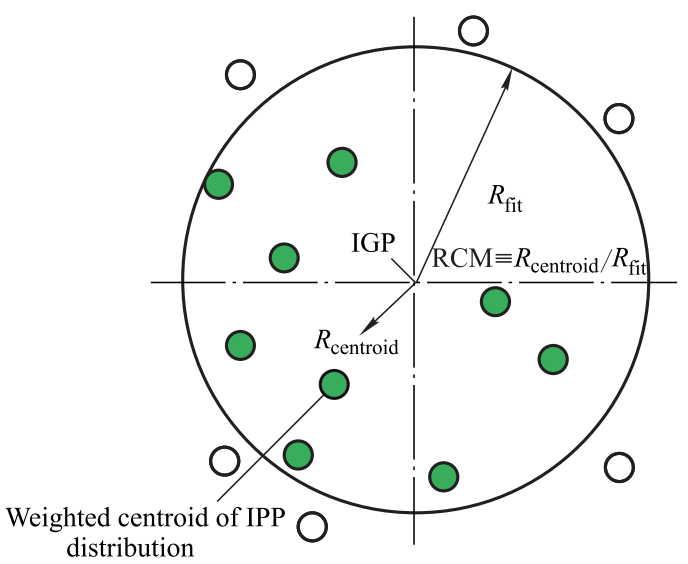

Fig. $2 R_{\text {fit }}$ and RCM describe IPPs distribution

In summary, when we construct an under-sampled spatial threat model, the objective is to compute the maximum estimation error of IPPs that could ever be observed, so that ionospheric delay estimation error will be fully enveloped. Thus, the value of the spatial threat model indicates the potential maximum uncertainty when IPPs are under-sampling or non-uniform distribution.

\section{Improvement of ionospheric spatial threat model}

\subsection{Problem statement}

As described above, the spatial threat model which is a function of fit radius and RCM is currently used in WAAS [25]. However, in the calculation process of the 2D threat model, if the measurements have the lower elevations, the IPPs in single-layer shell cannot fully reflect the real propagation path. In other words, when the elevation of the station's propagation path is relatively small, the RCM of the single-layer cannot fully reflect the effect of IPPs to IGP. The RCM in different heights of thin shell may be different. As shown in Fig. 3, this time cannot easily determine the height of a single-layer which is used as the calculation standard is more reasonable. 


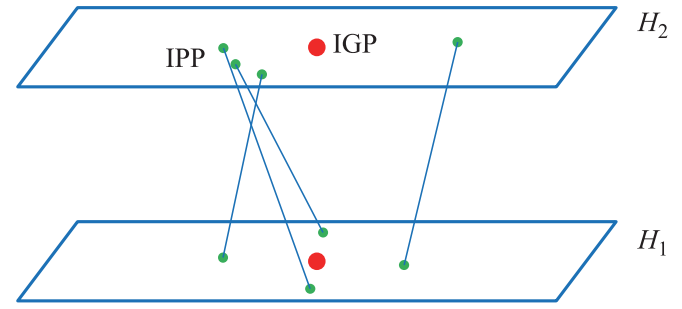

Fig. 3 IPPs spatial distribution

\subsection{An improved layered two-dimensional spatial threat model}

Based on the above analysis and the main deficiency of 2D spatial threat model, this paper develops an L2D spatial threat model combined with the $2 \mathrm{D}$ threat model and tomography theory that further reduces GIVE and improves usability on users as well as system service availability.

Consider that the double layer can characterize the basic IPPs distribution changes at each layer. Thus, the two RCMs index is used to modeling the L2D spatial threat model and the choice of them is mainly based on the weighting value which has more impact on the ionosphere. This means that the selection of the double layer is mainly based on the proportion of the different heights in the ionosphere. Because the electronic concentration of the ionospheric $\mathrm{F}$ layers changes more obvious (more than $130 \mathrm{~km}$ ), according to the description of ionospheric spatial structure based on Chapman function [15], consider the computational complexity and the ionospheric disturbance as well as the geometry of station satellite propagation path, we chose the $H_{1}$ layer as $350 \mathrm{~km}$ and $H_{2}$ as $450 \mathrm{~km}$. The formula Chapman function is

$$
C h\left(h, H_{k}\right)=\exp \left(1+\frac{h_{k}-h}{H_{k}}-\exp \left(\frac{h_{k}-h}{H_{k}}\right)\right) \text {. }
$$

In summary, we introduce a two RCMs index, and use three variables $R_{\text {fit }}, \mathrm{RCM}_{1}$ (calculated by $H_{1}$ layer) and $\mathrm{RCM}_{2}$ (calculated by $H_{2}$ layer) to describe the distribution characteristics of IPPs near the IGP and construct an L2D spatial threat model.

Thus the 2D threat model is extended, the calculation formula of the original value of the threat model is adjusted as follows:

$$
\begin{gathered}
\sigma_{\text {undersampled }}^{\text {raw }}\left(R_{\text {fit }}, \mathrm{RCM}_{1}, \mathrm{RCM}_{2}\right)= \\
\max _{\text {overk }, T}\left[\sqrt{\frac{\left|I_{k}-\hat{I}_{k}\right|^{2}}{K_{\text {undersampled }}^{2}}-\hat{\sigma}_{k}^{2}}\right]
\end{gathered}
$$

where the meaning of the parameters in (14) is the same as the $2 \mathrm{D}$ model.

\subsection{Result of spatial threat model}

During a quiet period, the under-sampling value of the threat model is equal to 0 . However, when the ionosphere at the storm period, the value is calculated by measurements with high disturbance coefficients to ensure the effectiveness of GIVE. The $K p$ index and the disturbance storm time (Dst) are publicly available indices of global geomagnetic activity, ionospheric delay measurements of continuous operational reference system (CORS) receivers under storm conditions during 2000 to 2005 (see Table 1) are selected to analyze and obtain the raw data of 2D and L2D ionospheric spatial threat model.

Table 1 Ionospheric storm days during 2000 to 2005

\begin{tabular}{cccc}
\hline Date & $K p$ & $\operatorname{Dst}(n T)$ & $\begin{array}{c}\text { Geomagnetic } \\
\text { storm class }\end{array}$ \\
\hline 2000.07 .15 & 9 & -295 & Extreme \\
2000.07 .16 & 8 & -288 & Severe \\
2001.03 .31 & 9 & -378 & Extreme \\
2001.11 .24 & 9 & -204 & Extreme \\
2003.10 .29 & 9 & -339 & Extreme \\
2003.10 .30 & 9 & -368 & Extreme \\
2003.10 .31 & 8 & -240 & Severe \\
2003.11 .20 & 9 & -402 & Extreme \\
2004.07 .27 & 9 & -152 & Extreme \\
2004.11 .08 & 9 & -359 & Extreme \\
2004.11 .10 & 9 & -264 & Extreme \\
2005.08 .24 & 9 & -191 & Extreme \\
2005.09 .11 & 9 & -131 & Extreme \\
\hline
\end{tabular}

We use RCM and $R_{\text {fit }}$ to characterize the distribution of IPPs. The obtained 2D threat model is shown in Fig. 4, and it represents the maximum residual for each pair of radius and RCM. It is logical to assume that as the threat model is attempting to protect against under-sampled ionospheric disturbances, the value of the threat model must be monotonically increasing in both radius and RCM [21]. Thus, the under-sampled estimation error $\sigma_{\text {undersampled increases }}$ with the increase of RCM and $R_{\text {fit }}$. The maximum value is chosen as the model current index value.

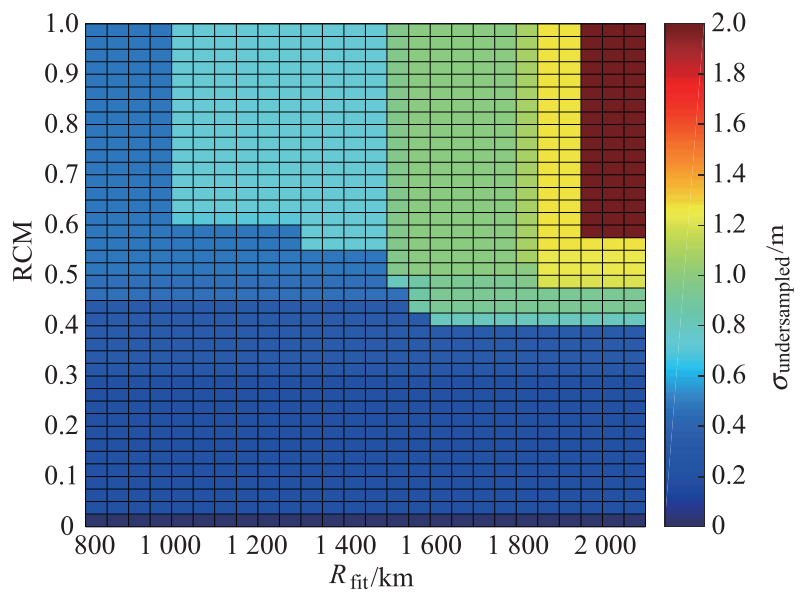

Fig. 4 Results of 2D spatial threat model 
Similarly, we use the double RCMs to construct the L2D spatial threat model and then the estimated residuals will increase with the $R_{\mathrm{fit}}, \mathrm{RCM}_{1}$ and $\mathrm{RCM}_{2}$. Fig. 5 shows a few typical calculated results of the L2D spatial threat model, and the $\mathrm{RCM}_{2}$ of the second layer $\left(H_{2}\right.$ layer) is used as the graph index. In other words, the downgrade of the L2D spatial model picture is equivalent to a $2 \mathrm{D}$ model

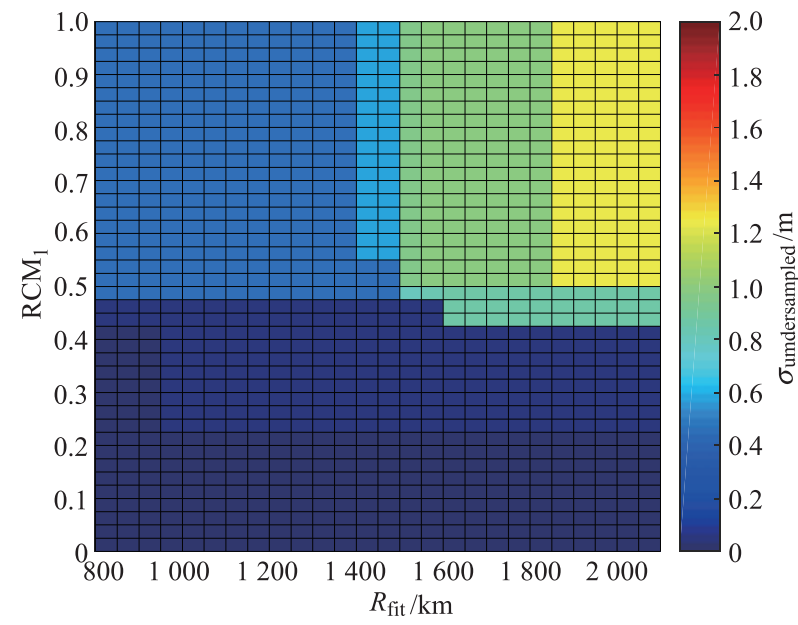

(a) Results of $\mathrm{RCM}_{2}=0.475$

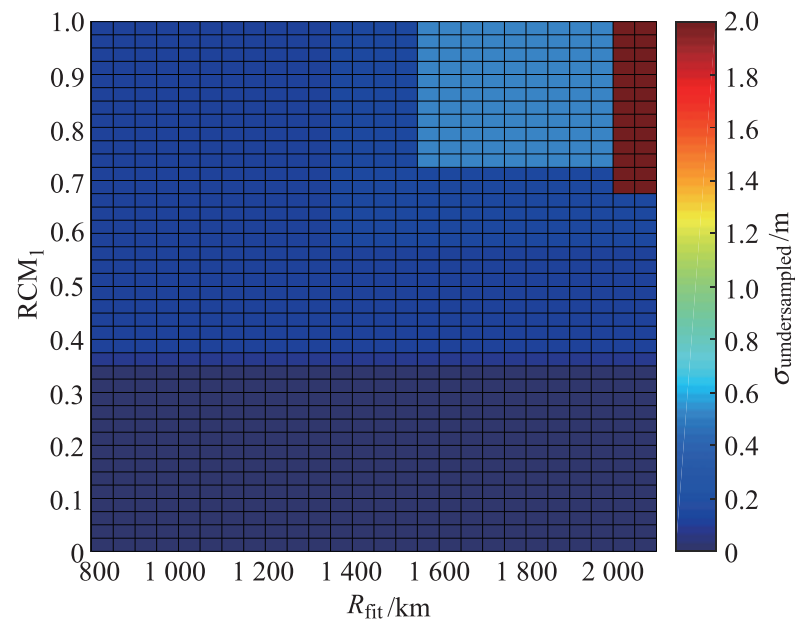

(c) Results of $\mathrm{RCM}_{2}=0.675$ and each picture in Fig. 5 can be understood as $H_{2}$ layer of $\mathrm{RCM}_{2}$ is a fixed value, and now the figure shows the values of under-sampled estimation error $\sigma_{\text {undersampled }}$ make regular changes with the $\mathrm{RCM}_{1}$ ( $H_{1}$ layer) and fit radius. From Fig. 4 and Fig. 5, a conclusion can be drawn that the L2D threat model is more accurate, and it reduces the conservative GIVEs of the 2D model.

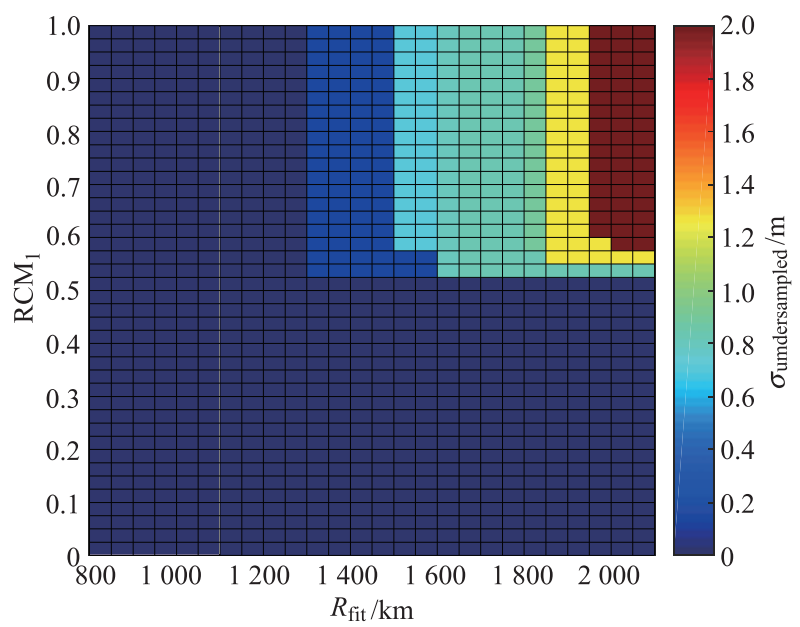

(b) Results of $\mathrm{RCM}_{2}=0.575$

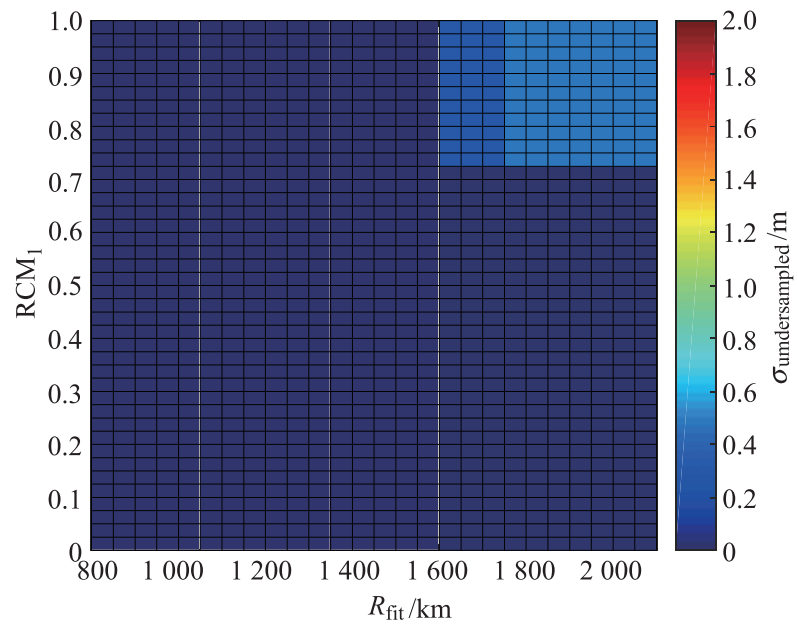

(d) Results of $\mathrm{RCM}_{2}=0.775$

Fig. 5 A few typical results of L2D spatial threat model

\section{Performance analysis}

In this paper, ionospheric delay measurements of WAAS receivers and CORS receivers are used to analyze the performance of the ionospheric L2D spatial threat model, including user grid correction analysis and system service performance analysis.

\subsection{User grid correction analysis}

SBAS broadcasts corrections of IGPs including GIVDs and GIVEs, the user receives those parameters and uses a linear interpolation algorithm to obtain their own ionospheric delay correction values and confidence bound corresponding to the satellites in sight and the confidence bounds from IGPs [30]. The calculation formula is

$$
\hat{I}_{\mathrm{user}}=\sum_{i=1}^{n} W_{i}\left(X_{\mathrm{IPP}}, Y_{\mathrm{IPP}}\right) \tilde{I}_{\mathrm{IGP}, i}
$$

$$
\sigma_{\mathrm{UIVE}}^{2}=\sum_{i=1}^{n} W_{i}\left(x_{\mathrm{IPP}}, y_{\mathrm{IPP}}\right) \cdot \sigma_{\text {ionogrid }, i}^{2}
$$

where $W_{i}\left(X_{\mathrm{IPP}}, Y_{\mathrm{IPP}}\right)$ is interpolation coefficient corre- 
sponding to adjacent grid nodes.

As shown in Fig. 6, we use the ionospheric delay measurements of 38 WAAS stations (red triangle) to build the 2D and L2D spatial threat models respectively, and select 15 CORS stations (green triangle) as the user station to verify biases between the dual bands true values of CORS and the calculated values.

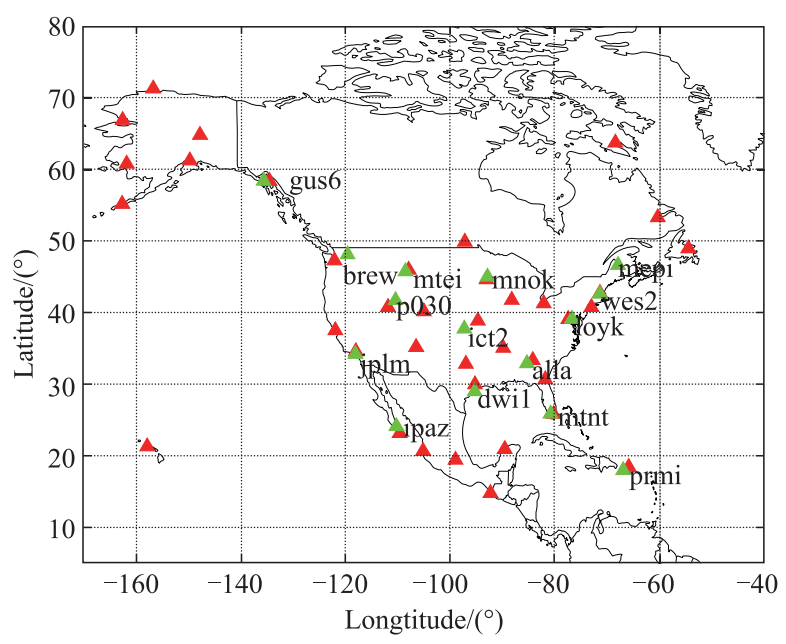

Fig. 6 WAAS and CORS stations receivers

According to the space environment prediction center (SEPC), the ionosphere has experienced quiet, moderately disturbed and severely disturbed ionospheric conditions during the whole March as shown in Fig. 7. The max planetary index of geomagnetic activity $K p$ index reaches 8 , and the disturbance has reached the level of geomagnetic storm. Thus, we can use the whole March of 2015 to analyze the performance of user grid correction and error bound in this section.

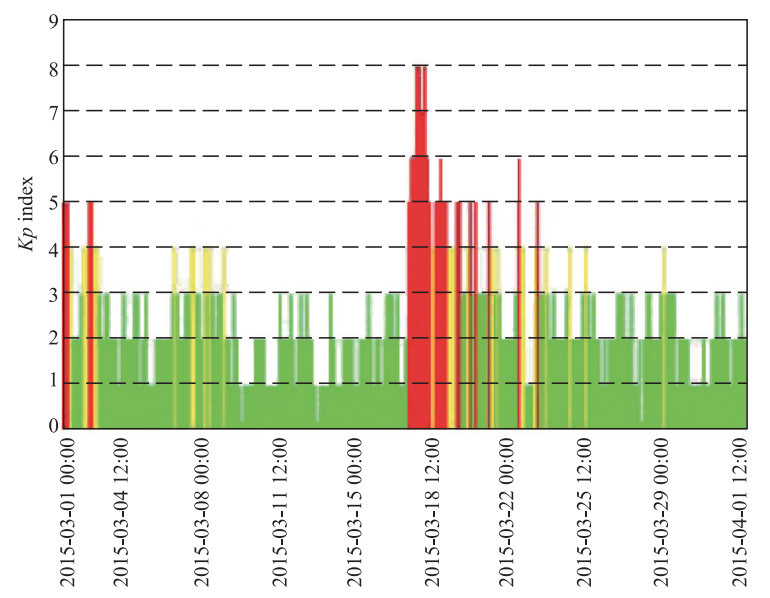

Fig. $7 \quad K p$ index during the whole March

Fig. 8 shows the result at brew station from satellite pseudo random noise (PRN) 23 at March 17, 2015. It can be obviously seen that both of the user ionospheric vertical error (UIVE) from the 2D and L2D spatial threat models can envelop the estimation error. However, under the premise of meeting the bounds, the UIVE of the L2D model is much smaller. To further illustrate the performance of the improved ionospheric spatial threat model, we give the statistical results of UIVEs mean reduction rate. The UIVEs mean reduction rate is

$$
\begin{gathered}
\eta=\frac{1}{n} . \\
\sum_{i=1}^{n} \frac{\mathrm{UIVE}_{i}\left(R_{\mathrm{fit}}, \mathrm{RCM}\right)-\mathrm{UIVE}_{i}\left(R_{\mathrm{fit}}, \mathrm{RCM}_{1}, \mathrm{RCM}_{2}\right)}{\mathrm{UIVE}_{i}\left(R_{\mathrm{fit}}, \mathrm{RCM}\right)} .
\end{gathered}
$$

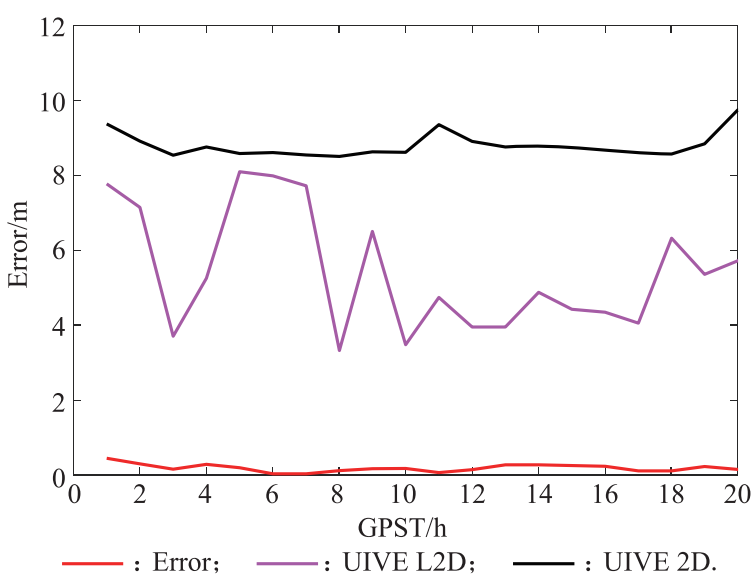

Fig. 8 Result of ionospheric delay grid correction

Table 2 shows the statistical results of user ionospheric delay grid correction in March 2015. Similar to the analysis of Fig. 8, during the calculation of the data for one month, the mean reduction rate reaches more than $16 \%$ from the L2D threat model with the effective UIVEs in the 15 CORS. This shows that, under the premise of satisfying the envelope, the L2D model for the user station can provide a more accurate GIVE value and it significantly improves the user's integrity and availability.

Table 2 Statistical results of UIVEs in March 2015

\begin{tabular}{cccc}
\hline User & \multicolumn{2}{c}{ UIVEs bound } & Mean reduction \\
\cline { 2 - 3 } site & $\begin{array}{c}\text { 2D spatial } \\
\text { threat model }\end{array}$ & $\begin{array}{c}\text { L2D spatial } \\
\text { threat model }\end{array}$ & $\begin{array}{c}\text { in UIVEs } \\
\text { alla }\end{array}$ \\
brew & 100 & 100 & 46.0 \\
dwi1 & 100 & 100 & 25.7 \\
gus6 & 100 & 100 & 47.3 \\
ict2 & 100 & 100 & 37.4 \\
ipaz & 100 & 100 & 47.4 \\
jplm & 100 & 100 & 29.6 \\
loyk & 100 & 100 & 31.1 \\
mepi & 100 & 100 & 43.6 \\
mnok & 100 & 100 & 34.2 \\
mtei & 100 & 100 & 34.2 \\
mtnt & 100 & 100 & 32.3 \\
p030 & 100 & 100 & 33.0 \\
prmi & 100 & 100 & 43.4 \\
wes2 & 100 & 100 & 16.1 \\
\hline
\end{tabular}




\subsection{System service performance analysis}

In this section, we will analyze the influence of the system service ability by using ionospheric delay correction.

Two methods in this paper will be used to evaluate and quantify GIVE from WAAS service areas of the United States, and then matlab algorithm availability simulation tool (MAAST) software is used to compute the horizontal protection level (HPL) and vertical protection level (VPL) of conterminous United States (CONUS). In order to ensure the reliability of the measurement, besides GIVEs using two threat models respectively discussed in this paper, the rest of integrity parameters is from WAAS broadcast.

Ionospheric delay measurements from 38 WAAS stations under quiet (20 June 2016, $K p=2$ ) and severely disturbed (17 March 2015, Kp=8) ionospheric conditions are selected to evaluate GIVE, and other WAAS broadcast integrity parameters are used to get the horizontal and vertical protection threshold of continental United States by MAAST software, as shown in Fig. 9 and Fig. 10.

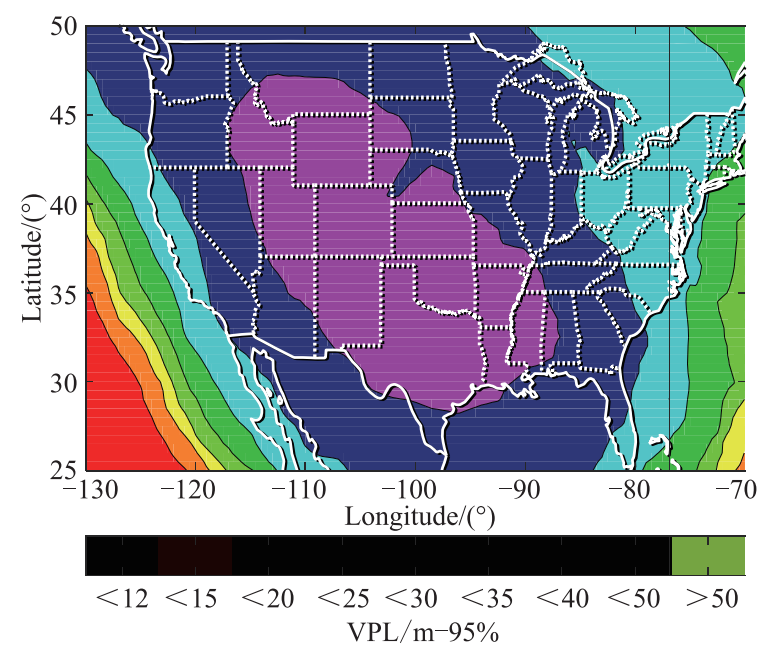

(a) Results of VPL based 2D threat model

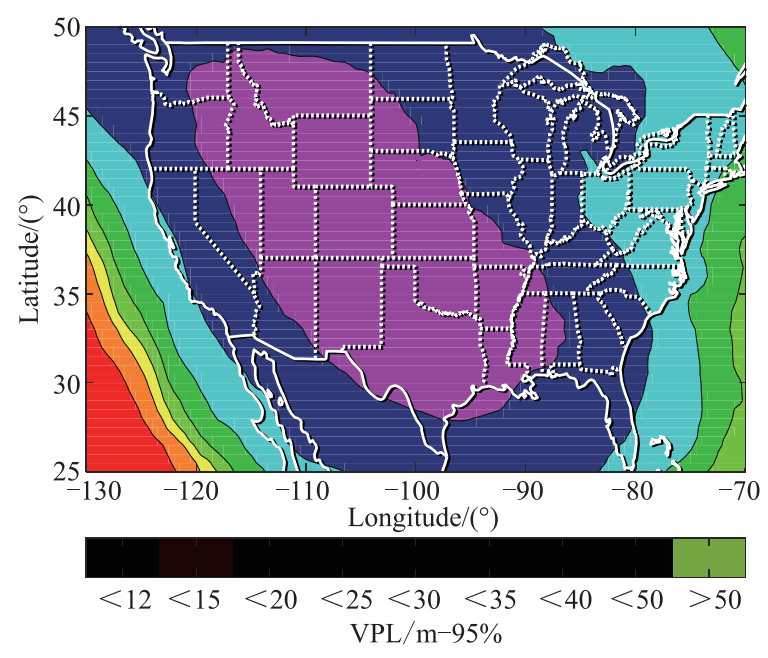

(b) Results of VPL based L2D threat model

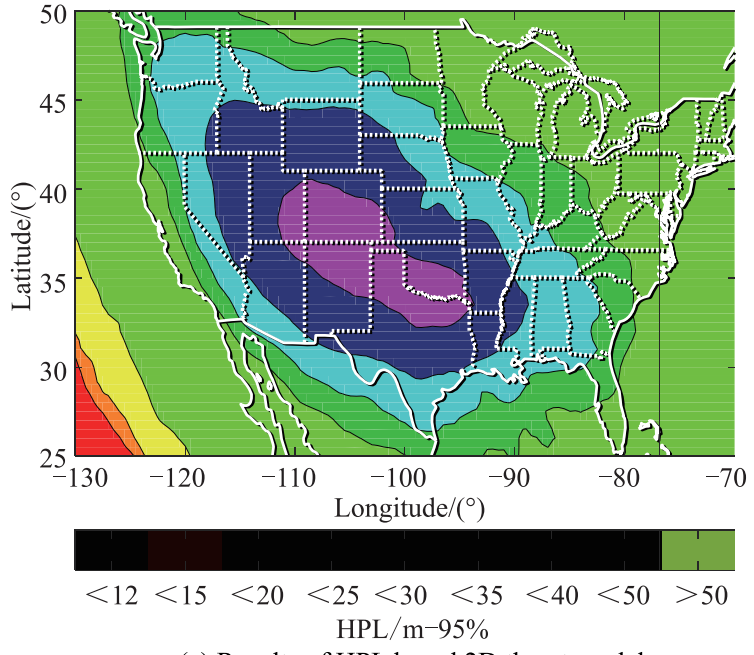

(c) Results of HPL based 2D threat model

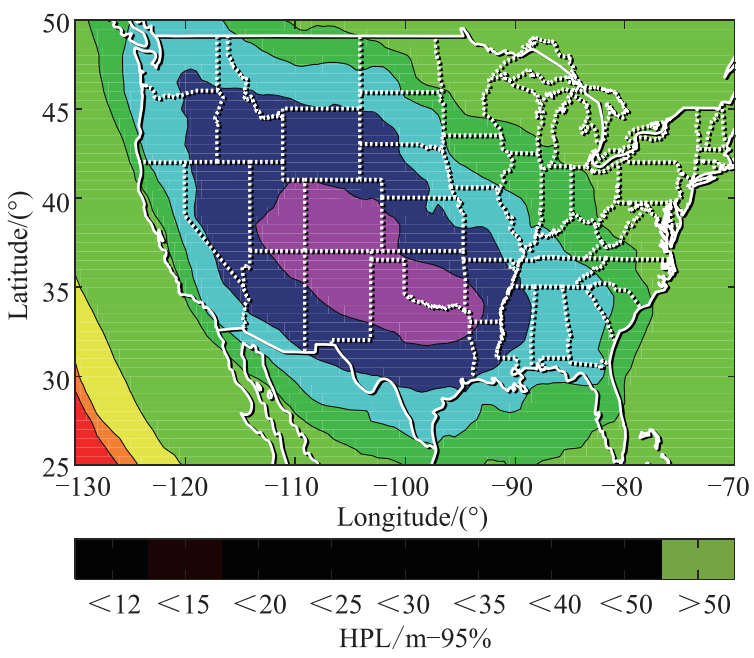

(d) Results of HPL based L2D threat model

Fig. 9 Results of HPL and VPL in CONUS on a disturbed day

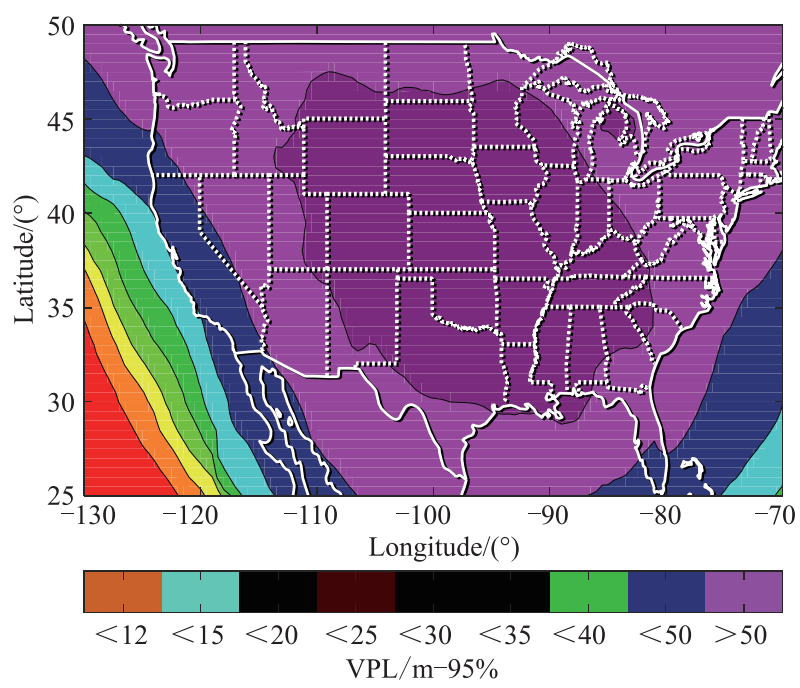

(a) Results of VPL based 2D threat model 


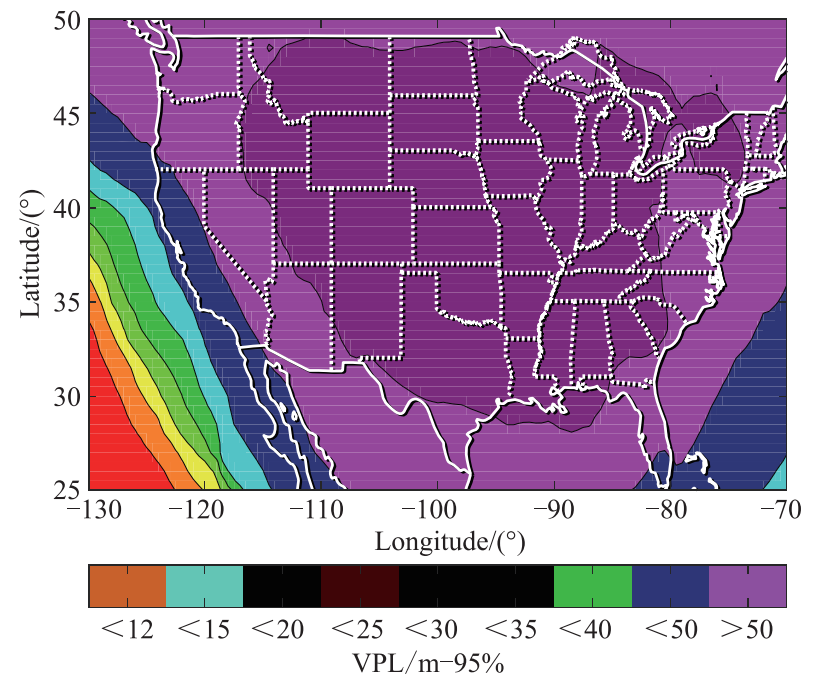

(b) Results of VPL based L2D threat model

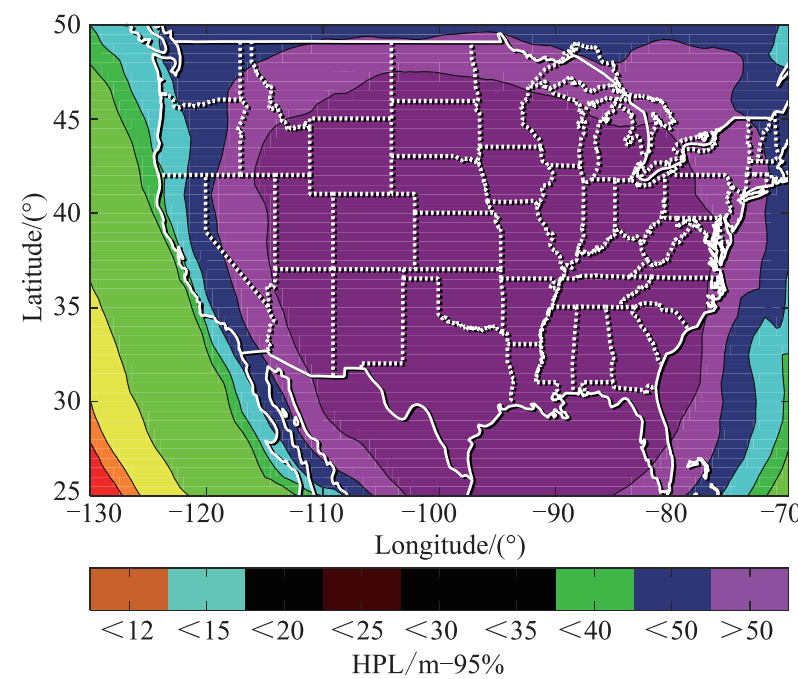

(c) Results of HPL based 2D threat model

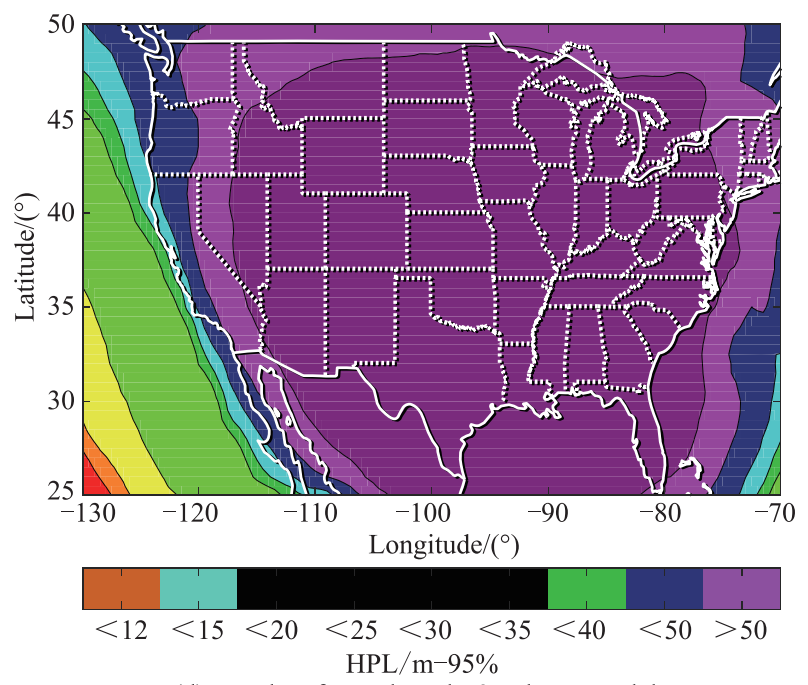

(d) Results of HPL based L2D threat model

Fig. 10 Results of HPL and VPL in CONUS on a quite day

It can be seen from Fig. 9 and Fig. 10 that both of the two algorithms protection thresholds are significantly greater than the quite period by the influence of the ionosphere storm, but the calculation protection threshold with double RCM parameters is less than the 2D threat model.

In Table $3, \eta_{\mathrm{VPL}}(5 \%)$ and $\eta_{\mathrm{HPL}}(5 \%)$ are the percentage of the CONUS, whose 95\% VPL and HPL from the L2D threat model have more than 5\% reduction rate relative to those VPL and HPL from the 2D threat model, respectively. It can be seen from Table 3 that using the L2D threat model, the $95 \%$ PLs of CONUS are $28 \%$, even under disturbed days, which reaches about $5 \%$ reduction rates. Thus, the L2D threat model reduces the PLs and achieves better system service performance.

Table 3 Statistical results of the PLs reduction percentages under different ionospheric conditions

\begin{tabular}{ccc} 
& & $\%$ \\
\hline Ionospheric condition & $\eta_{\mathrm{VPL}}(5 \%)$ & $\eta_{\mathrm{HPL}}(5 \%)$ \\
\hline quite (20 June 2016) & 36.8 & 43.4 \\
\hline $\begin{array}{c}\text { moderately disturbed } \\
\text { (13 October 2015) }\end{array}$ & 34.0 & 37.9 \\
\hline $\begin{array}{c}\text { severely disturbed } \\
\text { (17 March 2015) }\end{array}$ & 35.2 & 28.0 \\
\hline
\end{tabular}

\section{Conclusions}

The traditional threat model characterizes the ionosphere irregularity behavior by the RCM and fit radius. It is established on the $350 \mathrm{~km}$ reference plane defined by the SBAS system. However, this model fails to account for the spatial variation of the ionosphere, especially when the low elevation IPPs distribution deviation is larger. And furthermore, its calculated GIVEs and UIVEs value is too conservative and the availability of SBAS users will be suffered. Therefore, we consider the spatial variability of the ionosphere and propose an L2D spatial threat model with double RCMs based on the 2D model, reducing the GIVEs and enhancing the service capabilities of SBAS.

Simulation analysis results indicate that using the L2D spatial threat model to estimate GIVEs, the UIVEs of all user sites have more than $16 \%$ reduction rate. And the 95\% PLs of CONUS are $28 \%$, even under disturbed days, which reaches about $5 \%$ reduction rates. From the above, the tighter ionospheric delay estimation error can be obtained with the L2D spatial threat model which reduces UIVEs, and achieves better service performance.

\section{Acknowledgment}

We would like to thank the support of the Collaborative Innovation Center of Geospatial Technology.

\section{References}

[1] KIM J, LEE Y J. Using ionospheric corrections from the 
space-based augmentation systems for low earth orbiting satellites. GPS Solutions, 2015, 19(3): 423-431.

[2] RATNAM D V, DABBAKUTI J R K K, SUNDA S. Modeling of ionospheric time delays based on a multishell spherical harmonics function approach. IEEE Journal of Selected Topics in Applied Earth Observations and Remote Sensing, 2017, 10(12): $5784-5790$.

[3] SIVAVARAPRASAD G, RATNAM D V. Short-term forecasting of ionospheric total electron content over a low-latitude global navigation satellite system station. IET Radar Sonar \& Navigation, 2017, 11(8): 1309 - 1320.

[4] TAKAHASHI H, WRASSE C M, DENARDINI C M, et al. Ionospheric TEC weather map over South America. Space Weather-the International Journal of Research \& Applications, 2017, 14(11): $937-949$.

[5] GAMPALA S, DEVANABOYINA V R. Application of SST to forecast ionospheric delays using GPS observations. IET Radar Sonar \& Navigation, 2017, 11(7): 1070 - 1080.

[6] SPARKS L, KOMJATHY A, MANNUCCI A J. Sudden ionospheric delay decorrelation and its impact on the wide area augmentation system (WAAS). Radio Science, 2016, 39(1): $1-8$.

[7] RODRÍGUEZ-BOUZA M, PAPARINI C, OTERO X, et al. Southern European ionospheric TEC maps based on Kriging technique to monitor ionosphere behavior. Advances in Space Research, 2017, 60(8): 1606-1616.

[8] HUANG L, ZHANG H, XU P, et al. Kriging with unknown variance components for regional ionospheric reconstruction. Sensors, 2016, 17(3): 468-500.

[9] LAWRENCE S, BLANCH J, PANDYA N. Estimating ionospheric delay using Kriging: 1. Methodology. Radio Science, 2011, 46(6): 1-13.

[10] ABDELAZEEM M, ÇELIK R N, EL-RABBANY A. An accurate Kriging-based regional ionospheric model using combined GPS/BeiDou observations. Journal of Applied Geodesy, 2018, 12(1): $65-76$.

[11] LIU D, YU X, CHEN L, et al. Analysis on ionospheric delay variogram modeling in China. Proc. of the China Satellite Navigation Conference, 2017: 119-130.

[12] LEE J, KIM M. Optimized GNSS station selection to support long-term monitoring of ionospheric anomalies for aircraft landing systems. IEEE Trans. on Aerospace \& Electronic Systems, 2017, 53(1): 236-246.

[13] GRUNWALD G, BAKUŁA M, CIEĆKO A, et al. Examination of GPS/EGNOS integrity in north-eastern Poland. IET Radar Sonar \& Navigation, 2016, 10(1): 114-121.

[14] HAMEL P, SAMBOU D C, DARCES M, et al. Kriging method to perform scintillation maps based on measurement and GISM model. Radio Science, 2016, 49(9): 746-752.

[15] LIU P, LI R. Improving extended Kriging with chapman model and exponential variation function model. Lecture Notes in Electrical Engineering, 2016, v389: $177-187$.

[16] MINKWITZ D, VAN D B K G, GERZEN T, et al. Tomography of the ionospheric electron density with geostatistical inversion. Annales Geophysicae, 2015, 33(8): 1071-1079.

[17] MINKWITZ D, DEN BOOGAART K G, GERZEN T, et al. Ionospheric tomography by gradient-enhanced Kriging with STEC measurements and ionosonde characteristics. Annales Geophysicae, 2016, 34(11): 999-1010.

[18] EUGENE B. Considerations on ionospheric correction and integrity algorithm for Korean SBAS. Journal of Positioning, Navigation, and Timing, 2014, 3(1): 17-23.

[19] AMMANA S R, ACHANTA S D. Estimation of overbound on ionospheric spatial decorrelation over low-latitude region for ground-based augmentation systems. IET Radar Sonar \& Navigation, 2016, 10(3): 637-645.

[20] BORRIES C, JAKOWSKI N, KAURISTIE K, et al. On the dynamics of large-scale traveling ionospheric disturbances over Europe on 20 November 2003. Journal of Geophysical Research Space Physics, 2017, 122(1): 1199-1211.

[21] JUAN B, TODD W, PER E. Ionospheric threat model methodology for WAAS. Navigation, 2002, 49(49): 103-107.

[22] SAKAI T, MATSUNAGA K, HOSHINOO K, et al. Modeling ionospheric spatial threat based on dense observation datasets for MSAS. Proc. of the 21st International Technical Meeting of the Satellite Division of The Institute of Navigation, 2008: $1918-1928$.

[23] ONUR K M, YEGANEHSAHAB A, DURMAZ M. Estimation of GBAS ionospheric threat model parameters using continuous GNSS observations for category $i$ operations. Proc. of the EGU General Assembly Conference Abstracts. 2017: 13642.

[24] LEE J, PULLEN S, DATTA-BARUA S, et al. Real-time ionospheric threat adaptation using a space weather prediction for GNSS-based aircraft landing systems. IEEE Trans. on Intelligent Transportation Systems, 2017, 18(7): 1752-1761.

[25] BANG E, LEE J, LEE J, et al. Constructing ionospheric irregularity threat model for Korean SBAS. Proc. of the Ion Pacific PNT Meeting, 2013, 8900(6): 296-306.

[26] ZHANG Q, RUI L I, WANG Z. An improved ionospheric spatial threat model for SBAS. Chinese Journal of Electronics, 2017, 26(5): $1105-1110$.

[27] LIU D, FENG J, CHEN L, et al. A study on construction of ionospheric spatial threat model for China SBAS. Proc. of the China Satellite Navigation Conference, 2017: 195-208.

[28] BLANCH J. Using Kriging to bound satellite ranging errors due to the ionosphere. 2004.

[29] DATTA-BARUA S. Ionospheric threats to the integrity of airborne GPS users. 2008.

[30] DO-229E. Minimum operational performance standards for global positioning system / satellite-based augmentation system airborne equipment. Washington, D.C.: Radio Technical Commission for Aeronautics, 2016.

\section{Biographies}

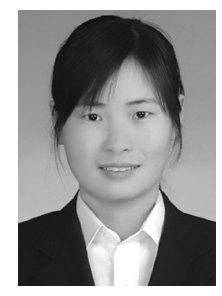

BAO Junjie was born in 1989. She is a Ph.D. candidate in communications and information systems at School of Electronic and Information Engineering, Beihang University. Her current research is targeted on the technology of satellite-based augmentation system integrity algorithms, mainly ionospheric correction.

E-mail: baojunjie@buaa.edu.cn

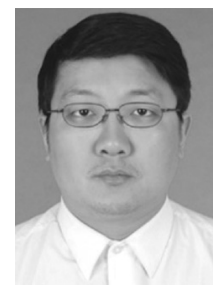

LI Rui was born in 1976. He received his Ph.D. degree from Beihang University in 2006. He is now a senior engineer in the School of Electronic and Information Engineering, Beihang University. His main interests cover required navigation performance and global navigation satellite system augmentation technologies in satellite-based augmentation system, ground based augmentation system, and aircraft based augmentation system.

E-mail: lee_ruin@263.net 


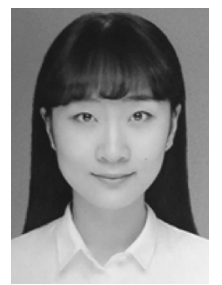

LIU Pan was born in 1993. She received her M.S. degree from Beihang University in 2017. Her research interest focuses on the technology of ionospheric correction.

E-mail: liupanlzu@yeah.net

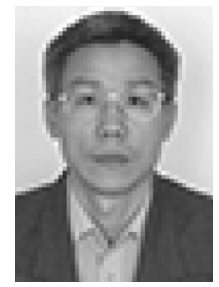

HUANG Zhigang was born in 1962. He received his Ph.D. degree from Beihang University in 2004. $\mathrm{He}$ is currently a professor in the School of Electronics and Information Engineering, Beihang University. His research interests include integrity algorithms and related software for ground based augmentation system and satellite-based augmentation system.

E-mail: baahzg@163.com 